\title{
High-Rate Charging Induced Intermediate Phases and Structural Changes of Layer- Structured Cathode for Lithium-Ion Batteries
}

\author{
Y.-N. Zhou \\ Submitted to Advanced Energy Materials
}

August 2016

\author{
Chemistry Department
}

Brookhaven National Laboratory

\author{
U.S. Department of Energy \\ [DOE Office of Science]
}

Notice: This manuscript has been authored by employees of Brookhaven Science Associates, LLC under Contract No. DE- SC0012704 with the U.S. Department of Energy. The publisher by accepting the manuscript for publication acknowledges that the United States Government retains a non-exclusive, paid-up, irrevocable, world-wide license to publish or reproduce the published form of this manuscript, or allow others to do so, for United States Government purposes. 


\section{DISCLAIMER}

This report was prepared as an account of work sponsored by an agency of the United States Government. Neither the United States Government nor any agency thereof, nor any of their employees, nor any of their contractors, subcontractors, or their employees, makes any warranty, express or implied, or assumes any legal liability or responsibility for the accuracy, completeness, or any third party's use or the results of such use of any information, apparatus, product, or process disclosed, or represents that its use would not infringe privately owned rights. Reference herein to any specific commercial product, process, or service by trade name, trademark, manufacturer, or otherwise, does not necessarily constitute or imply its endorsement, recommendation, or favoring by the United States Government or any agency thereof or its contractors or subcontractors. The views and opinions of authors expressed herein do not necessarily state or reflect those of the United States Government or any agency thereof. 


\section{High-Rate Charging Induced Intermediate Phases and Structural Changes of Layer- Structured Cathode for Lithium-Ion Batteries}

Yong-Ning Zhou, Ji-Li Yue, Enyuan Hu, Hong Li, Lin Gu*, Kyung-Wan Nam, Seong-Min Bak, Xiqian Yu, Jue Liu, Jianming Bai, Eric Dooryhee, Zheng-Wen Fu*, Xiao-Qing Yang*

Prof. Y.-N. Zhou

Department of Materials Science, Fudan University, Shanghai 200433, China

Prof. Y.-N. Zhou, Dr. E. Hu, Dr. S.-M. Bak, Dr. X. Yu, Dr. J. Liu, Dr. X.-Q. Yang

Department of Chemistry, Brookhaven National Laboratory, Upton, New York 11973, United

States

E-mail: xyang@bnl.gov

Mr. J.-L. Yue, Prof. Z.-W. Fu

Shanghai Key Laboratory of Molecular Catalysts and Innovative Materials, Department of

Chemistry \& Laser Chemistry Institute, Fudan University, Shanghai 200433, China

E-mail: zwfu@fudan.edu.cn

Prof. H. Li, Prof. L. Gu,

Laboratory for Advanced Materials \& Electron Microscopy, Beijing National Laboratory for

Condensed Matter Physics, Institute of Physics, Chinese Academy of Sciences, Beijing

100190, China

E-mail: l.gu@aphy.iphy.ac.cn

Prof. K.-W. Nam

Department of Energy and Materials Engineering, Dongguk University-Seoul, Seoul 100-715, Republic of Korea

Dr. J. Bai, Dr. E. Dooryhee

National Synchrotron Light Source II, Brookhaven National Laboratory, Upton, New York 11973, United States

Keywords: lithium ion batteries, cathode materials, high rate, structure change

Using fast time-resolved in situ X-ray diffraction, charge-rate dependent phase transition processes of layer structured cathode material $\mathrm{LiNi}_{1 / 3} \mathrm{Mn}_{1 / 3} \mathrm{Co}_{1 / 3} \mathrm{O}_{2}$ (NMC) for lithium-ion batteries are studied. During first charge, intermediate phases emerge at high rates of 10C, $30 \mathrm{C}$ and $60 \mathrm{C}$, but not at low rates of $0.1 \mathrm{C}$ and $1 \mathrm{C}$. These intermediate phases can be continuously observed during relaxation after the charging current was switched off. After half-way charging at high rate, sample studied by scanning transmission electron microscopy shows Li-rich and Li-poor phases coexistence with tetrahedral occupation of $\mathrm{Li}$ in $\mathrm{Li}$-poor phase. The high rate induced over-potential is thought to be the driving force for the 
formation of this intermediate Li-poor phase. The in situ quick X-ray absorption results show that the oxidation of $\mathrm{Ni}$ accelerates with increasing charging rate and the $\mathrm{Ni}^{4+}$ state can be reached at the end of charge with 30C rate. These results give new insights in the understanding of the layered cathodes during high-rate charging.

\section{Introduction}

In the efforts of expanding lithium-ion batteries (LIBs) from widely used in consumer electronics to large-scale applications such as electric vehicles, hybrid electrical vehicles and clean energy storage ${ }^{[1,2]}$, the rate capability and energy density are two very important issues. In order to improve the rate capability of the electrode materials in battery operation, practical approaches have been reported, such as using nanoparticle composed electrode materials and surface coatings to reduce ion diffusion length and increase electronic conductivity ${ }^{[3-5]}$. Although relationships between the high-rate capabilities and nanometer-size effects as well as chemical compositions and structures of nano-structured materials have been studied ${ }^{[6-10]}$, the fundamental understanding about the structural changes of the electrode materials during high rate cycling in real time is quite limited, partly due to experimental difficulties in ultrafast data collection requirement under operando conditions.

Recently, metastable structures (a continuous solid solution phase, rather than the two-phase separation) in nano-sized $\mathrm{LiFePO}_{4}$ (LFP) electrodes were captured experimentally during high-rate cycling using synchrotron based time-resolved X-ray diffraction (XRD) techniques $^{[11]}$. The results were analyzed and interpreted as non-equilibrium state for LFP during fast delithiation process. It is very interesting to see that the phase transition behavior of the electrode materials at high rate cycling is quite different from that at the low rate, as reported earlier (see literature ${ }^{[12,13]}$ about the metastable transient crystal phase in $\mathrm{Li}_{x} \mathrm{FePO}_{4}$ observed by time-resolved XRD). Furthermore, a new "lithium staging” structure of $\mathrm{Li}_{0.5} \mathrm{FePO}_{4}$ was observed by an aberration-corrected annular-bright-field scanning 
transmission electron microscopy (STEM) technique ${ }^{[14]}$. Although this Li-staging phase was not observed in situ, and the cycling rate was not high, the direct observation of the existence of intermediate phase with atomic resolution is very important for understanding the complicated structural changes of cathode materials during cycling.

The advantage of XRD is the large penetration depth of the photon probe into the sample and the statistical reliability due to averaging over the whole sample area illuminated by Xrays. The disadvantage is the limitation on new phase identification caused by peak broadening, due to the short coherence length (crystal grain size, not particle size) and/or large distribution of atomic inter-layer distances. Unfortunately, such short coherence length (as newly formed crystal) and lattice parameter distribution (newly formed crystal grains with varying lattice parameters) are very likely to occur during high rate cycling and XRD alone may not be able to give us a full picture. On the other hand, scanning transmission electron microscopy (STEM) is a great complementary technique to $\mathrm{XRD}^{[15]}$. It is capable to catch new crystal structures even with very small quantity and very short coherence length with atomic resolution. The disadvantage of STEM is the lack of statistical reliability since the sample area being probed is usually too small. Therefore, using the combination of XRD and STEM techniques will provide a more detailed full picture about the structural changes of the materials being studied. In addition, in situ quick X-ray absorption spectroscopy (Q-XAS) is another powerful technique for studying the high rate behavior of electrode materials ${ }^{[16]}$. It has the capability to distinguish the charge compensation contribution by different transition metal ions in the electrode materials during high rate charge-discharge, reflected by their valence state and local environment changes, such as in local symmetry and coordination numbers.

Inspired by the interesting studies introduced above, we carried out a structural study using the in situ time-resolved XRD and Q-XAS, as well as ex situ STEM on a commercially important $\mathrm{LiNi}_{1 / 3} \mathrm{Mn}_{1 / 3} \mathrm{Co}_{1 / 3} \mathrm{O}_{2}$ (NMC) cathode material during and after high rate cycling. 
The reason of selecting this particular NMC material is based on the importance of this material in today's commercial Li-ion batteries (much more widely used than LFP) and the totally different crystal structure from LFP (layer structured for NMC vs. olivine structured for LFP). In addition NMC exhibits a more complex phase diagram with different phase transition pathways during charge and discharge, when compared with LFP. The particle size of the NMC samples in this study is in the micron range (particle size: 0.8-1.0 $\mu$ m, Figure S1), which is the optimized particle size in the commercial Li-ion batteries using NMC materials. As we know, the crystal structure of the layer structured cathodes such as $\mathrm{LiCoO}_{2}, \mathrm{LiNiO}_{2}$, $\mathrm{LiNi}_{0.5} \mathrm{Mn}_{0.5} \mathrm{O}_{2}$ and $\mathrm{LiNi}_{1 / 3} \mathrm{Mn}_{1 / 3} \mathrm{Co}_{1 / 3} \mathrm{O}_{2}$ generally undertake a "solid solution - two-phase reaction - solid solution” phase transition pathway during the first charge process ${ }^{[17-32]}$. Two hexagonal $\mathrm{O} 3$ phases ( $\mathrm{H} 1$ and $\mathrm{H} 2$ ) are usually involved for the normal charging range (the formation of the $\mathrm{O} 1$ phase resulted from high voltage charging should be avoid for good cycle performance). It is well known that layer structured cathode materials $\mathrm{LiMO}_{2}(\mathrm{M}=\mathrm{Ni}$, Co, $\mathrm{Mn}$ ) have better rate capability comparing with olivine LFP systems ${ }^{[9,10]}$, due to their higher electronic conductivity, higher lithium ion diffusion coefficient and two-dimensional diffusion paths. However, to the best of our knowledge, not much systematic studies have been reported on the phase transition behavior of the layer structured cathode materials during high current rate charging. In the present work, the structural changes of NMC in a wide range of current rates (from $0.1 \mathrm{C}$ to 60C) charging are systematically studied using synchrotron based time-resolved in situ XRD. The contribution from each transition metal ions ( $\mathrm{Ni}, \mathrm{Mn}, \mathrm{Co}$ ) to charge compensation on charging is obtained by in situ Q-XAS. The details of atomic arrangement and local phase distributions for NMC samples harvested from half-way charged cell at 30C rate are studied by the aberration-corrected STEM technique. An intermediate phase is observed using in situ time resolved XRD during the first charging cycle when current rates higher than 10C are applied. 


\section{Results and Discussion}

$\mathrm{LiNi}_{1 / 3} \mathrm{Mn}_{1 / 3} \mathrm{Co}_{1 / 3} \mathrm{O}_{2}$ (NMC) has a typical $\mathrm{NaFeO}_{2}$-type layered structure with a space group of R-3m. Detailed information about the crystal structure is shown in Figure S2 and Table S1. By using specially designed in situ cells and optimized X-ray diffraction technique for high rate characterization, the in situ XRD patterns of the NMC cathode were captured during the first charge at different current rates of $0.1 \mathrm{C}, 1 \mathrm{C}, 10 \mathrm{C}, 30 \mathrm{C}$ and $60 \mathrm{C}$ (the $\mathrm{C}$ rate is calculated based on a specific capacity of $160 \mathrm{mAh} \mathrm{g}^{-1}$ ). In order to collect data with good signal-tonoise ratio and $2 \theta$ angle resolution in a very short time, most data were collected in a narrow $2 \theta$ angle range near the 003 reflection only. Figure 1 shows the 003 diffraction peak evolution of $\mathrm{Li}_{1-x} \mathrm{Ni}_{1 / 3} \mathrm{Mn}_{1 / 3} \mathrm{Co}_{1 / 3} \mathrm{O}_{2}$ cathode from $x=0$ to $x=0.7$ ( $x$ value is calculated from the charging curve, $x=0$ is the pristine sample and $x=0.7$ is considered as the fully charged sample. Charge curves during the in situ XRD experiments are shown in Figure S3). At 0.1C, a typical "solid solution - two phase - solid solution" phase transition behavior is observed, involving two hexagonal phases (H1 and H2). This agrees well with the previous in situ XRD study via synchrotron X-ray ${ }^{[22,30-32]}$. Some research groups ${ }^{[23-29]}$ also studied the structure change of NMC during initial charge process via ex situ XRD by lab X-ray, but they did not reveal the two-phase coexsistence region. We believe this is because of the low angle resolution and time resolution of the ex situ lab XRD, compared with in situ synchrotron XRD. Very interestingly, when the $\mathrm{C}$ rate increases to 10C, a new, broad peak emerges between the $\mathrm{H} 1$ and $\mathrm{H} 2$ phases with a low intensity at 10C, and becomes more pronounced at 30C and 60C, indicating the formation of intermediate phases. Comparing to the metastable phase in LFP system ${ }^{[12,13]}$, the intermediate phases in NMC system cover a much wider range of $2 \theta$ angles and $x$ values bridging the $\mathrm{H} 1$ and $\mathrm{H} 2$ phases. This could be caused by either the solidsolution behavior or the multi-phase nature of the intermediate phases, and very likely by both. It is worth noting that the appearance of the new $\mathrm{H} 2$ phase and the disappearance of the initial $\mathrm{H} 1$ phase occur at an earlier stage of charging process (lower $x$ values) when the $\mathrm{C}$ rate is 
increased. This high rate accelerated phase transition is very interesting. The high rate charging can create high inhomogeneity of Li content, which in turn could enhance the new phase formation. On the other hand, the extra driving force provided by the over-potential effect of high rate should be helpful in overcoming the energy barrier for phase transition. Such extra driving force together with the high inhomogeneity may also play very important roles in forming the intermediate structures, as will be discussed later using the STEM results. Full XRD patterns of NMC over an extended range of $2 \theta$ angles and at a high charge rate (30C) are shown in Figure S4. They agree well with the diffractogram of Figure 1 and the signature of the intermediate phase can be observed for all the Bragg peaks, although the $2 \theta$ angle peak separation is a little poor due to the short wavelength used. It should be noted that for $60 \mathrm{C}$ charge, the peak is broader than those for low rate charge at the high potential range. This indicates that high rate charging creates high inhomogeneity of Li content, resulting in a wider distribution of $c$ lattice parameter at the high potential range. In addition, NMC is fully converted into the $\mathrm{H} 2$ phase at the end of charge, even at 60C rate charge, indicating the excellent rate capability. In comparison, the LFP at a rate of 10C charge still shows residues of the pristine LFP phase at the fully charged state ${ }^{[11-13]}$.

Figure 2 shows the contour plot of the 003 diffraction peak of $\mathrm{Li}_{1-x} \mathrm{Ni}_{1 / 3} \mathrm{Co}_{1 / 3} \mathrm{Mn}_{1 / 3} \mathrm{O}_{2}$ as a function of time during a 55 second charge $(x \sim 0.35)$ at a current rate of 30C and then relaxed over a time period of 480 seconds when no charging current applied. In order to see the changes during relaxation more clearly, the detailed in situ XRD patterns in three different time zones are also plotted in Figure 2. At the moment the charging current is just cut off, relatively sharp $\mathrm{H} 1$ and $\mathrm{H} 2$ peaks and a broad intermediate phase peak can be clearly identified. With increasing relaxation time in the first 200 seconds, the H1 peak gradually disappears, the intermediate phase peak broadens and the $\mathrm{H} 2$ peak shifts to higher $2 \theta$ angles and sharpens. Between 200 and 480 seconds, the H2 peak changes course from sharpening to broadening and ends up as a quite broad peak at 480 seconds. At the same time, in the broad 
peak region representing the intermediate phase, two or three peaks are gradually developed, as indicated by the yellow areas in the counter plot. These results show that the structural relaxation after high rate charging is a quite complicated process. The high rate charging creates very steep Li concentration gradients and large inhomogeneities from the surface to the center of the particles, as well as a large number of structural defects. With the help of extra driving force provided by the higher over-potential, multiple intermediate phases with different Li contents and lattice parameter $c$ can be nucleated and grown. During relaxation, with the rearrangement of $\mathrm{Li}$ ions, some of the newly nucleated intermediate phases can continuously grow up. It should be noted that these intermediate phases can be maintained several hours, even several days after the charging current was cut off, well beyond the $480 \mathrm{~s}$ time period shown in Figure 2. This is different from the relaxation behavior of the metastable structure in some LFP systems, where the lifetime was estimated to be $\sim 30 \min ^{[12]}$. In fact, the intermediate phases should be relaxed to the thermodynamically stable phase after certain time. Unfortunately, we did not follow the relaxation process to the end, since we were more interested in how long it can be maintained to do our ex situ TEM study. Our results suggest the developed intermediate phases can be maintained more than several days to give us enough time to do the TEM study. Taking advantage of these relatively stable intermediate phases in the half way charged NMC samples $(x \sim 0.35)$, we were able to carry out ex situ STEM studies on these intermediate phases with atomic level resolution.

In order to visualize the structural changes of NMC caused by high rate charge, pristine NMC and samples harvested from cells after 30C charge were studied using the STEM technique. More than 20 images were collected for each sample at different spots to get better statistics, but only a few representative ones with good quality are presented here. The results of pristine NMC are shown in Figure 3. Figure 3a presents a schematic of the atom arrangement along [010] projection, which is the best direction for observing the layer structured cathode material, since separated columns of $\mathrm{Li}, \mathrm{O}$, and transition metal $(\mathrm{TM}=\mathrm{Ni}$, 
Co, and $\mathrm{Mn}$ ) ions are aligned. An extensive number of atomic resolution high-angle annular dark-field (HAADF) and annular-bright-field (ABF) STEM images of NMC were obtained and two representative images are shown in Figure $3 \mathrm{~b}$ and c. It should be noted that the contrast varies as $Z^{1.7}$ where " $Z$ ” is the atomic number for the HAADF images, but varies as $Z^{1 / 3}$ for the ABF images. Since HAADF is more sensitive to the heavy atoms, transition metal (TM) ions (3b sites) are clearly observed from the HAADF image in bright dots in Figure 3b, while the lighter atoms of $\mathrm{Li}$ (3a sites) and $\mathrm{O}$ (6c sites) are showed more clearly in the ABF image (Figure 3c). Figure 3d shows the line contrast profile of TM columns along the [42ㅍ] direction projected at the [010] zone axis. It demonstrates that pristine NMC exhibits a welldefined layered structure, and the positions of TM (3b sites) are well resolved at the atomic

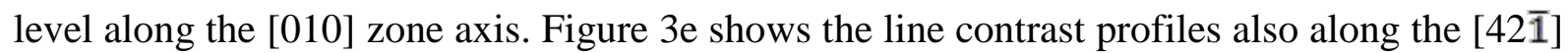
direction. It shows a very uniform distribution of the "TM-O-Li-O-TM" ordering, indicating a perfect layered structure without observable Li-TM anti-site defects, which are frequently observed in Ni containing layer-structured cathode materials ${ }^{[33]}$.

To observe the intermediate phases of NMC formed by high rate charging at the atomic scale, ex situ STEM images were collected on the relaxed sample (48 hour relaxation) after half-way charge $(x \sim 0.35)$ at 30C rate. The typical HAADF and ABF images taken along the [110] zone axes are shown in Figure 4. The uniformly distributed TM layers can be seen from the HAADF image (Figure 4a, b and c), which is identical to the TM layers in pristine NMC (Figure 3b). It indicates that the structure of TM layers is well preserved after half-way charge at high rate. On the other hand, the $\mathrm{ABF}$ image taken at the same sample position shows two different types of domains in the green and red dashed squares in Figure $4 \mathrm{~d}$, and in more details in Figure 4e and $\mathrm{f}$. The location of the Li columns in the green dashed square region in Figure 4e is different from that in the red dashed square region in Figure 4f. In Figure 4f, the Li columns are no longer located in the middle position of two TM columns (actually, it should be in the middle of two oxygen columns, but the TM layers have much stronger 
contrast). The middle position corresponds to the typical octahedral sites as shown in pristine sample. It looks like the lithium ions in Figure $4 \mathrm{f}$ are located at the pseudo-tetrahedral sites, as identified by the deviation away from the middle point of two adjacent TM layers. From the previous study ${ }^{[34]}$, it is known that the Li diffusion path in the layer structured cathode materials during charge-discharge is an "octahedral - tetrahedral - octahedral” movement pathway driven by a divacancy hopping mechanism along the $a-b$ plane. During high-rate charging, the higher over-potential can provide extra driving force for the motion of lithium ions, causing a largely inhomogeneous lithium ion distribution and structural defects within the sample particles. It is assumed that the tetrahedral site occupation becomes energetically favorable for $\mathrm{Li}$ in some $\mathrm{Li}$-poor regions and the intermediate phases are formed with such occupation. Actually, lithium ions could be stabilized at the tetrahedral sites in some layerstructured cathodes as reported in the literature for $\mathrm{T} 2-\mathrm{Li}_{x} \mathrm{CoO}_{2}(0.52<x<0.72)$ and T2$\mathrm{Li}_{2 / 3} \mathrm{Ni}_{1 / 3} \mathrm{Mn}_{2 / 3} \mathrm{O}_{2}{ }^{[35,36]}$. From the STEM images, it is very likely that some lithium ions occupy the tetrahedral sites as shown in the schematic in Figure S5. The occurrence of regions with different contents and occupations of $\mathrm{Li}$ distributions might be the origin of the intermediate phase observed with XRD (as explained in the previous section). In addition the HAADF and ABF images collected on a fully charged $(x=0.7)$ sample are shown in Figure S6. The layered structure is still well preserved in the bulk, but some Li-TM anti-site occupation (3a and 3b sites) can be observed at the surface region. Remaining Li ions mainly occupy the octahedral sites in the middle of two oxygen slabs, and no more intermediate phase domains are observed. This is consistent with the single phase H2 observed by XRD for the fully charged sample.

The valence state and local environment of each transition metal element in NMC are monitored by in situ quick X-ray absorption technique. To avoid the lag of reaction near the window part of the cell ${ }^{[37]}$, glassy carbon is used to enhance the pressure of the window of the in situ cells. Figure 5a-c shows the changes of the X-ray absorption near edge spectroscopy 
(XANES) at the Ni, Co and Mn K-edges during 30C charge, respectively. The K-edge of Ni shifts continuously to higher energy, indicating the oxidation of $\mathrm{Ni}$ ions from $\mathrm{Ni}^{2+}$ to $\mathrm{Ni}^{4+}$ during the charging process. In contrast, the K-edges of Co and Mn exhibit almost no energy shift. The Co and Mn K-edge features change shape, indicating that the local environment changes around Co and $\mathrm{Mn}$ ions, but $\mathrm{Co}$ and $\mathrm{Mn}$ do not contribute much to the charge compensation. Same results are obtained at $1 \mathrm{C}$ and 10C rates presented in Figure S7. These results suggest that charge compensation during charging mainly arises from Ni. Charge compensation is not affected much by the different $\mathrm{C}$ rates. Figure $5 \mathrm{~d}$ shows the edge shift of Ni K-edge XANES as functions of the state of charge ( $x$ values) at different $\mathrm{C}$ rates. Interestingly, comparing the rate effects at the same charge state (same $x$ value), the oxidation state of $\mathrm{Ni}$ ions is higher at faster charging rates, especially in the half-way charge state $(x \sim$ 0.35). If all the charges passed to the sample were equally used to extract $\mathrm{Li}$, and $\mathrm{Ni}$ is the only charge compensation contributor, the oxidation state of $\mathrm{Ni}$ should be linear to the $x$ value and independent to the charge rate. In fact, these three curves are not linear and also charge rate dependent, showing that the effectiveness of Li extraction is different at different charge states and also rate dependent. The plotted higher oxidation state of $\mathrm{Ni}$ at the same nominal $x$ value actually means that when the total charge passed are the same, the higher rate has higher efficiency to extract more Li (higher $x$ value) than the lower rates. This is also consistent with the in situ XRD results shown in Figure 2. Since no rigid K-edge shift for Mn and Co during charge, the information shown in Figure $5 \mathrm{~d}$ for Ni cannot be obtained for $\mathrm{Mn}$ and Co. From the Fourier-transformed extended X-ray absorption fine structure (FT-EXAFS) spectra shown in Figure S8, the most noticeable spectral changes occur at the Ni-edge, indicating the significant changes of coordination shell and bond length around Ni during high rate charging. This is in a good agreement with the XANES results as well as with our previous study ${ }^{[38]}$.

Based on the data above, we unveil the real-time phase transition behavior of layer structured NMC cathode during fast charging. Intermediate phases are first observed when a 
$10 \mathrm{C}$ rate is applied, which become more pronounced at higher charging rates of 30C and 60C. They bridge the $\mathrm{H} 1$ and $\mathrm{H} 2$ phases. It can be assumed that high rate induced large overpotential could provide extra driving force to overcome the energy barrier for the nucleation of the intermediate phases and facilitate the multi-phase reactions. From the STEM results, we observe abnormal regions with tetrahedral $\mathrm{Li}$ ion occupation (Li poor region), in addition to the normal regions with octahedral $\mathrm{Li}$ occupation ( $\mathrm{Li}$ rich region) in samples at half-way charged state $(x=0.35)$ after high rate charging. The lattice parameter $c$ of the Li poor region is larger than that of the Li rich region. In contrast, for the pristine $(x=0)$ or fully charged sample $(x=0.7)$, only one type of region with octahedral Li occupation is observed. Theoretical calculations of the Li occupation in layer structured cathode materials showing that the free-energy variation of octahedral and tetrahedral Li occupation in relation to the distance between adjacent oxygen layers was reported in the literature ${ }^{[39]}$. It was suggested that the activation energy for tetrahedral occupation becomes lower than that for octahedral occupation, when the inter-layer distance between adjacent oxygen layers of Li increases to a certain value (2.9 $\AA$ for $\mathrm{LiCoO}_{2}$ ). This suggests that the preferred occupation sites for Li could be the tetrahedral site, when the such inter-layer distance is larger than a certain value. Table S2 shows the measured lattice parameters of the phases observed from STEM images. It can be seen that the parameter $c$ in Figure $4 \mathrm{f}$ is larger than that in Figure 4c. This agrees well with the prediction of the published calculation study ${ }^{[39]}$.

In situ quick X-ray absorption spectra provide useful information about the contribution of each transition metal element $(\mathrm{Ni}, \mathrm{Co}, \mathrm{Mn})$ to the charge compensation at different $\mathrm{C}$ rates. The fully oxidation of $\mathrm{Ni}$ from $\mathrm{Ni}^{2+}$ to $\mathrm{Ni}^{4+}$ during charge, even at a high rate of $30 \mathrm{C}$, indicates the electrochemical reaction and ion diffusion within the particles of NMC is rather fast. Ni oxidation is faster and more effective than that of Co and $\mathrm{Mn}$, when higher rate charging is applied. This is in good agreement with the calculated lower activation barrier surrounding $\mathrm{Ni}^{2+}(210 \mathrm{meV})$ comparing with $\mathrm{Co}^{3+}(310 \mathrm{meV})$ and $\mathrm{Mn}^{4+}(340 \mathrm{meV})$ for $\mathrm{Li}$ 
motion in the layer structure cathode systems ${ }^{[40]}$. These results provide an important guidance for designing NMC for high rate cycling: higher Ni content in NMC could be beneficial to high rate capability, as long as the negative effects of structural stability and cation mixing caused by high Ni content can be well managed.

\section{Conclusion}

In summary, we present the exploratory study of the non-equilibrium phase transition behavior in layer structured NMC cathode system during high rate cycling. It provides direct evidence for the different phase transition behaviors than those during low rate charging. Intermediate phases were observed at high rate charging at 10C, 30C, and 30C by in situ XRD. In contrast, such intermediate phase formation was not observed during low rate charging. Since the formation of intermediate phases is strongly related to the larger over-potential generated by high rate charging, during the low rate charge, the over-potential may not be high enough to facilitate the formation of intermediate phases. Phase coexistence heterogeneities and tetrahedral occupation of $\mathrm{Li}$ in the Li-poor phase, which could be one of the intermediate phases were observed in the half-way charged NMC using ex situ STEM. By using the in situ quick X-ray absorption technique, we find that the charge compensation contribution by $\mathrm{Ni}$ ions is faster and more effective at the higher $\mathrm{C}$ rate, suggesting the benefit of higher Ni content in NMC for rate capability. These results show that the high rate induced intermediate phase formation process reported in the literature for olivine structured LFP system, also exist in layer structured NMC system. This, raises the question about the presence of such intermediate states in other systems, such as layer structured $\mathrm{LiCoO}_{2}$ and cubic spinel structured $\mathrm{LiMn}_{2} \mathrm{O}_{4}$. These important results will further inspire theoretical and experimental studies on the high rate induced structural changes for a wide spectrum of cathode materials. 


\section{Experimental Section}

Samples and cell test: The $\mathrm{LiNi}_{1 / 3} \mathrm{Co}_{1 / 3} \mathrm{Mn}_{1 / 3} \mathrm{O}_{2}$ cathode materials were provided by an industrial partner. The electrode was prepared by slurring the active material, carbon black, and polyvinlidenefluoride (PVDF) with a weight ratio of 70:14:16 in N-methyl-2-pyrrolidone (NMP) solvent, then coating the mixture onto aluminum foil current collectors. The electrode was dried at $120^{\circ} \mathrm{C}$ for 12 hours, and then punched into electrode disks (16 mm in diameter). The loading of the active material on the electrode was about $2 \mathrm{mg} \mathrm{cm}^{-2}$. Testing cells were assembled into coin cells in an argon-filled glove box. High-purity lithium foil was used as the anode. $1 \mathrm{M} \mathrm{LiPF}_{6}$ in ethylene carbonate (EC) and dimethyl carbonate (DMC) solvent (3:7 by volume) was used as electrolyte. Galvanostatic charge-discharge measurements were carried out at room temperature on a Land CT 2001A battery test system.

In situ XRD characterization: Most of the in situ X-ray diffraction patterns were collected at beamline X14A of the National Synchrotron Light Source (NSLS) at Brookhaven National Laboratory (BNL) by a linear position sensitive silicon detector. The wavelength used was $0.7747 \AA$. The region of interest was limited around the 003 reflection. In situ XRD patterns with Bragg peaks covering (003) to (113) at 30C rate was collected at beamline XPD of the National Synchrotron Light Source II (NSLS-II) at BNL by a large-area, flat panel detector. The XRD pattern of the pristine NMC was collected at beamline X7B of NSLS with a X-ray wavelength of $0.3196 \AA$ A. Specially designed electrochemical coin cells using kapton film windows on both sides were employed for in situ.

In situ Quick XAS characterization: The Q-XAS experiment was performed at beamline X18A of the National Synchrotron Light Source (NSLS, BNL). The in situ Mn, Co and Ni Kedge spectra were collected separately in the transmission mode. About 16-17 XAS spectra were recorded in a 30 second time interval, with the best achievable temporal resolution of 2 seconds per scan. 8 successive spectra were merged into one to get a better signal-to-noise ratio. Therefore, the real temporal resolution in this work is about 15 seconds. Energy 
calibration was carried out using the first inflection point of the reference spectrum of metal foils which were simultaneously collected during each measurement during the in situ experiments.

STEM characterization: STEM specimens were prepared by sonication of the charged cathode in anhydrous dimethyl carbonate and drop-coating the materials individually onto carbon lacey films and sealed in airtight bottles before immediately transfer into the STEM column. A JEM-ARM200F STEM operated at $200 \mathrm{kV}$ and equipped with double aberrationcorrectors for both probe-forming and imaging lenses was used to perform high-angle annular-dark-field (HAADF) and annular-bright-field (ABF) imaging. The attainable spatial resolution of the microscope is $78 \mathrm{pm}$ at the incident semiangle of $25 \mathrm{mrad}$. To observe $\mathrm{Li}$ directly using ABF collection geometry, the acceptance semiangle in this study was fixed between 12 and 25 mrad. The STEM ABF and HAADF images were taken simultaneously at the optimal defocus value of the HAADF imaging condition, which was more defocused than the optimal ABF imaging condition on this instrument. Thus, the contrast in the ABF image is reversed with the bright area corresponding to the atomic positions.

\section{Supporting Information}

Supporting Information is available from the Wiley Online Library or from the author.

\section{Acknowledgements}

Y.N.Z. and J.L.Y. contributed equally to this work. The work at Brookhaven National Laboratory was supported by the U.S. Department of Energy, the Assistant Secretary for Energy Efficiency and Renewable Energy, Office of Vehicle Technologies under Contract No. DE-SC0012704. Use of the National Synchrotron Light Source (NSLS) and of the National Synchrotron Light Source II (NSLS-II), Brookhaven National Laboratory, was supported by the U.S. Department of Energy, Office of Science, Office of Basic Energy Sciences, under 
Contracts No. DE-AC02-98CH10886 and No. DE-SC0012704, respectively. The work at Fudan University and Institute of Physics, Chinese Academy of Sciences was supported by the NSFC (No.51502039 and No.U1430104), 1000 Youth Talents Plan and Science \& Technology Commission of Shanghai Municipality (08DZ2270500 and 11JC1400500). The authors thank technical supports by the beamline scientists at X14A and X18A of NSLS, and at the XPD beamline of NSLS-II at Brookhaven National Laboratory. We gratefully acknowledge the help by beamline scientists Sungsik Lee and Benjamin Reinhart at 12BM of Advanced Photon Source at Argonne National Laboratory, supported by the U.S. Department of Energy, Basic Energy Science, under Contract No. DE-AC02-06CH11357.

Received: ((will be filled in by the editorial staff))

Revised: ((will be filled in by the editorial staff)) Published online: ((will be filled in by the editorial staff))

[1] Kang, B.; Ceder, G. Nature 2009, 458, 190.

[2] Bruce, P. G.; Scrosati, B.; Tarascon, J. M. Angew. Chem. Int. Edit. 2008, 47, 2930.

[3] Zhang, H. G.; Yu, X. D.; Braun, P. V. Nat. Nanotechnol. 2011, 6, 277.

[4] Liu, C.; Gillette, E. I.; Chen X.; Pearse A. J.; Kozen A. C.; Schroeder M. A.; Gregorczyk K. E.; Lee, S. B.; Rubloff, G. W. Nat. Nanotechnol. 2014, 9, 1031.

[5] Wang, J. J.; Yang, J.; Tang, Y.; Liu, J.; Zhang, Y.; Liang, G.; Gauthier, M.; ChenWiegart, Y. K.; Banis, M. N.; Li, X.; Li, R.; Wang, J.; Sham, T. K.; Sun, X. Nat. Commun. 2014, 5, 3415.

[6] Okubo, M.; Hosono, E.; Kim, J.; Enomoto, M.; Kojima, N.; Kudo, T.; Zhou, H.; Honma, I. J. Am. Chem. Soc. 2007, 129, 7444.

[7] Shen, L. F.; Zhang, X. G.; Uchaker, E.; Yuan, C. Z.; Cao, G. Z. Adv. Energy Mater. 2012, 2, 691.

[8] Wang, H. L.; Yang, Y.; Liang, Y.; Cui, L. F.; Casalongue, H. S.; Li, Y.; Hong, G.; Cui, Y.; Dai, H. Angew. Chem. Int. Edit. 2011, 50, 7364. 
[9] Jo, M.; Hong, Y. S.; Choo, J.; Cho, J. J. Electrochem. Soc. 2009, 156, A430.

[10] Wu, S. L.; Zhang, W.; Song, X.; Shukla, A. K.; Liu, G.; Battaglia, V.; Srinivasan, V. J. Electrochem. Soc. 2012, 159, A438.

[11] Liu, H.; Strobridge, F. C.; Borkiewicz, F. C.; Borkiewicz, O. J.; Wiaderek, K. M.; Chapman, K. W.; Chupas, P. J.; Grey, C. P. Science 2014, 344, 1480.

[12] Orikasa, Y.; Maeda, T.; Koyama, Y.; Murayama, H.; Fukuda, K.; Tanida, H.; Arai, H.; Matsubara, E.; Uchimoto, Y.; Ogumi, Z. J. Am. Chem. Soc. 2013, 135, 5497.

[13] Orikasa, Y.; Maeda, T.; Koyama, Y.; Murayama, H.; Fukuda, K.; Tanida, H.; Arai, H.; Matsubara, E.; Uchimoto, Y.; Ogumi, Z. Chem. Mater. 2013, 25, 1032.

[14] Gu, L.; Zhu, C.; Li, H.; Yu, Y.; Li, C.; Tsukimoto, S.; Maier, J.; Ikuhara, Y. J. Am. Chem. Soc. 2011, 133, 4661.

[15] Yankovich, A. B.; Berkels, B.; Dahmen, W.; Binev, P.; Sanchez, S. I.; Bradley, S. A.; Li, A.; Szlufarska, I.; Voyles, P. M. Nat. Commun. 2014. 5, 4155.

[16] Yu, X. Q.; Yang, Q.; Zhou, Y. N.; Li, H.; Yang, X. Q.; Nam, K. W.; Ehrlich, S. N.; Khalid, S.; Meng, Y. S. Chem. Commun. 2012, 48, 11537.

[17] Reimers, J. N.; Dahn, J. R. J. Electrochem. Soc. 1992, 139, 2091.

[18] Li, W.; Reimers, J. N.; Dahn, J. R. Solid State Ionics 1993, 67, 123.

[19] Yang, X. Q.; Sun, X.; McBreen, J. Electrochem. Commun. 2000, 2, 100.

[20] Yang, X. Q.; Sun, X.; McBreen, J. Electrochem. Commun. 1999, 1, 227.

[21] Yang, X. Q.; McBreen, J.; Yoon, W. S.; Grey, C. P. Electrochem. Commun. 2002, 4, 649.

[22] Yoon, W. S.; Chung, K. Y.; McBreen, J; Yang, X. Q. Electrochem. Commun. 2006, 8, 1257.

[23] Zhou, Y. N.; Ma, J.; Hu, E.; Yu, X.; Gu, L.; Nam, K. W.; Chen, L.; Wang, Z.; Yang, X. Q. Nat. Commun. 2014, 5, 5381.

[24] Robert, R.; Bunzli, C.; Berg, E. J.; Novak, P. Chem. Mater. 2015, 27, 526. 
[25] Yabuuchi, N.; Makimura, Y.; Ohzuku, T. J. Electrochem. Soc. 2007, 154, A314.

[26] Yabuuchi, N.; Ohzuku, T. J. Power Sources 2003, 119, 171.

[27] Yabuuchi, N.; Koyama, Y.; Nakayama, N.; Ohzuku, T. J. Electrochem. Soc. 2005, 152, A1434.

[28] Choi, J.; Manthiram, A. J. J. Electrochem. Soc. 2005, 152, A1714.

[29] Yin, S. C.; Rho, Y. H.; Swainson, I.; Nazar, L. F. Chem. Mater. 2006, 18, 1901.

[30] Wise, A. M.; Ban, C.; Weker, J. N.; Misra, S.; Cavanagh, A. S.; Wu, Z.; Li, Z.; Whittingham, M. S.; Xu, K.; George, S. M.; Toney, M. F. Chem. Mater. 2015, 27, 6146.

[31] Conry, T. E.; Mehta, A.; Cabana, J.; Doeff, M. M. Chem. Mater. 2012, 24, 3307.

[32] Li, J.; Shunmugasundaram, R.; Doig, R.; Dahn, J. R. Chem. Mater. 2016, 28, 162.

[33] Rougier, A.; Gravereau, P.; Delmas, C. J. Electrochem. Soc. 1996, 143, 1168.

[34] Van der Ven, A.; Ceder, G. Electrochem. Solid State Lett. 2000, 3, 301.

[35] Carlier, D.; Croguennec, L.; Ceder, G.; Menetrier, M.; Shao-Horn, Y.; Delmas, C. Inorg. Chem. 2004, 43, 914.

[36] Paulsen, J. M.; Donaberger, R. A.; Dahn, J. R. Chem. Mater. 2000, 12, 2257.

[37] Strobridge, F.; Orvananos, B.; Croft, M.; Yu, H. C.; Robert, R.; Liu, H.; Zhong, Z.; Connolley, T.; Drakopoulos, M.; Thornton, K.; Grey, C. P. Chem. Mater. 2015, 27, 2374.

[38] Yu, X. Q.; Lyu, Y.; Gu, L.; Wu, H.; Bak, S. M.; Zhou, Y.; Amine, K.; Ehrich, S. N.; Li, H.; Nam, K. W.; Yang, X. Q. Adv. Energy Mater. 2014, 4, 1300950.

[39] Kang, K.; Ceder, G. Phys. Rev. B 2006, 74, 094105.

[40] Kang, K.; Meng, Y. S.; Breger, J.; Grey, C. P.; Ceder, G. Science 2006, 311, 977. 


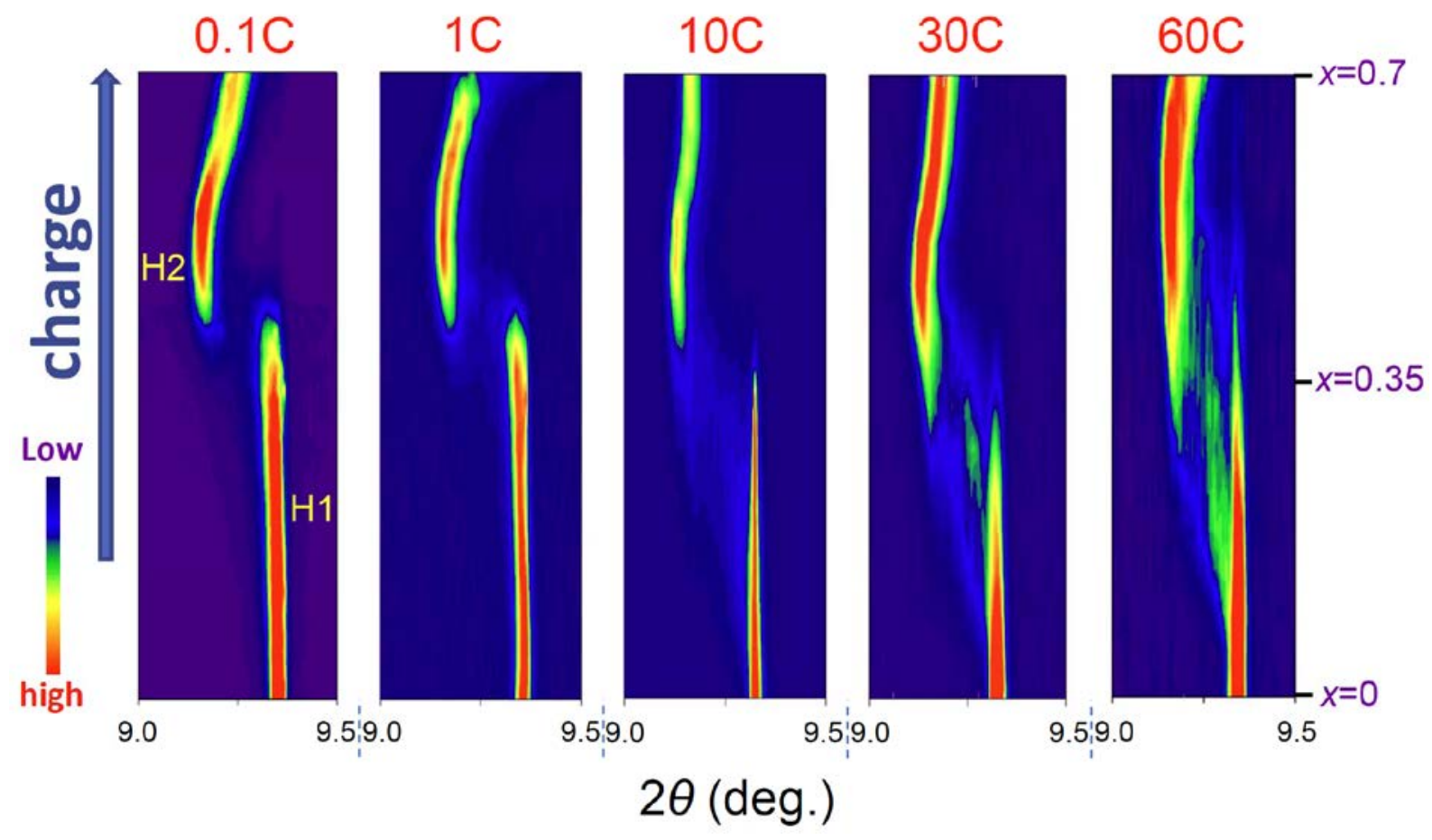

Figure 1. In situ XRD of NMC during the first charge. Contour plot of the 003 diffraction peak of $\mathrm{Li}_{1-x} \mathrm{Ni}_{1 / 3} \mathrm{Co}_{1 / 3} \mathrm{Mn}_{1 / 3} \mathrm{O}_{2}$ with increasing $x$ between $x=0$ and $x=0.7$ during the first charge process at different $\mathrm{C}$ rates $(0.1 \mathrm{C}, 1 \mathrm{C}, 10 \mathrm{C}, 30 \mathrm{C}, 60 \mathrm{C})$. Data were collected at X14A at NSLS with a wavelength of $0.7747 \AA$. 


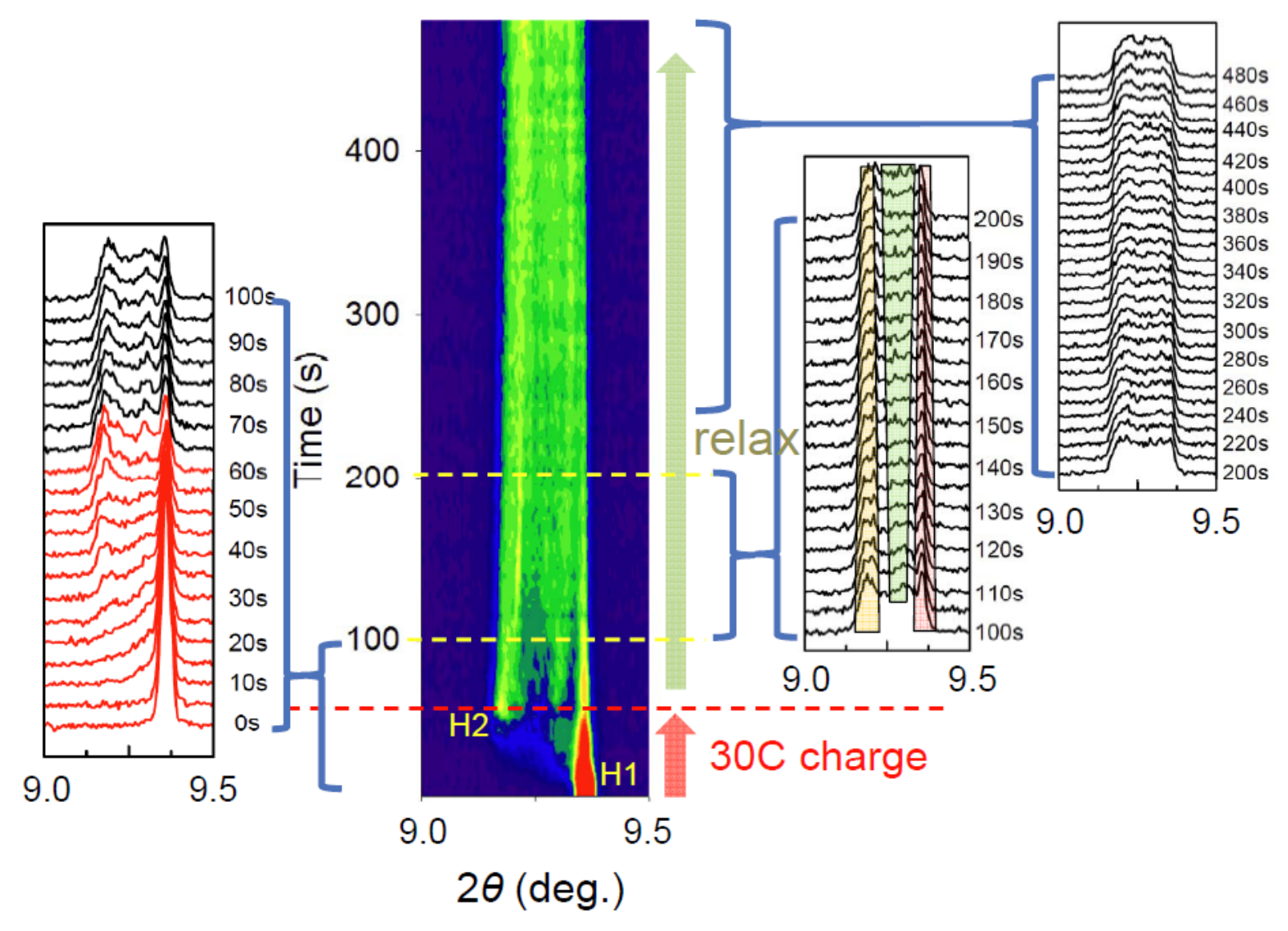

Figure 2. Structure change during relaxation process. Contour plot of the 003 diffraction peak as a function of charge and relaxation time. Detailed in situ XRD patterns of $\mathrm{Li}_{1}$ ${ }_{x} \mathrm{Ni}_{1 / 3} \mathrm{Co}_{1 / 3} \mathrm{Mn}_{1 / 3} \mathrm{O}_{2}$ are shown for three different time zones during relaxation after 55 second charging $(x=0.35)$ at a current rate of 30C. 


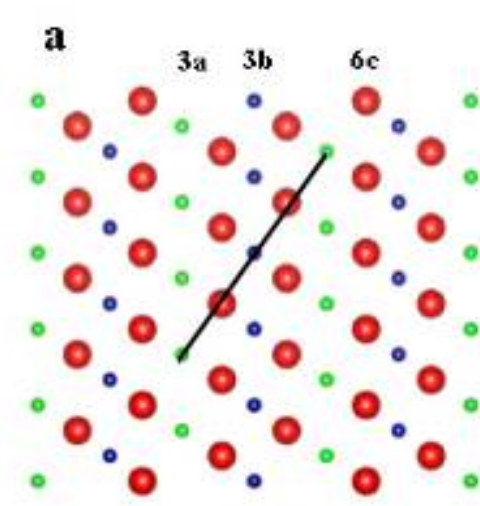

b
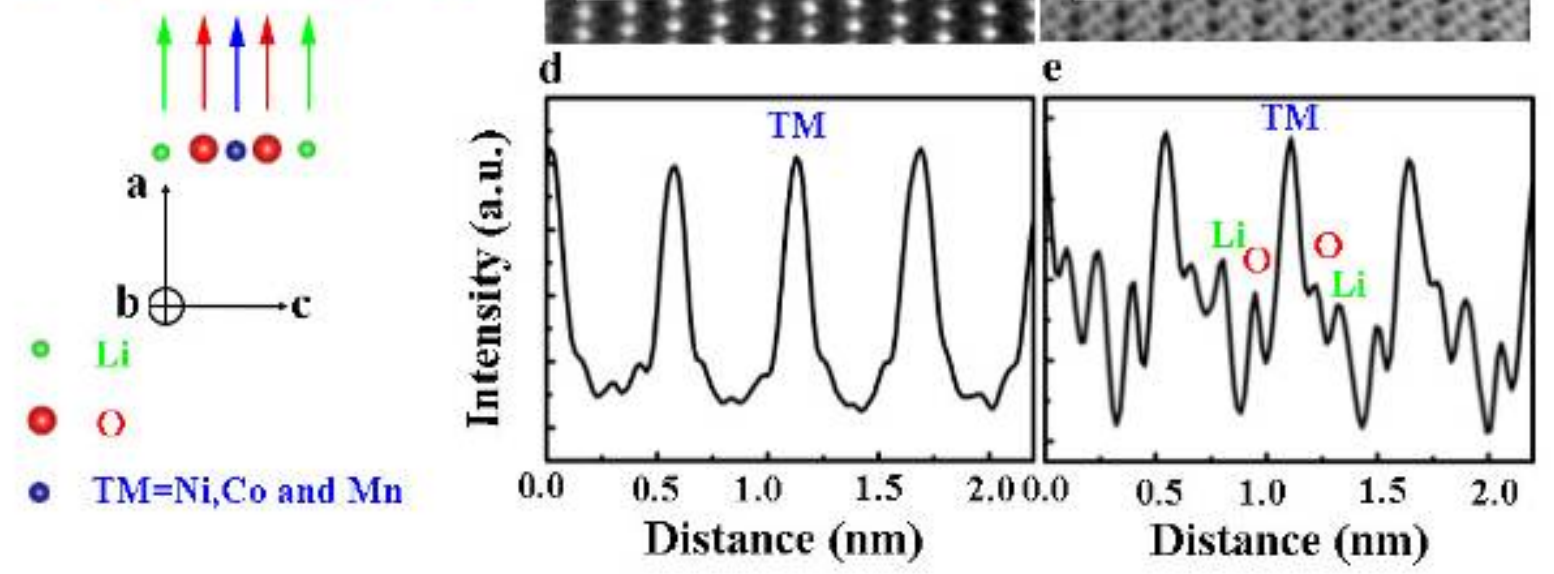

Figure 3. Structure of pristine NMC materials. a) A schematic of lattice structure, b) HAADF and c) ABF STEM images of pristine NMC from the [010] projection. The corresponding line profiles of d) TM (TM = Ni, Co and $\mathrm{Mn}$ ) atoms in HAADF image and e) $\mathrm{TM}, \mathrm{Li}$ and $\mathrm{O}$ atoms in $\mathrm{ABF}$ image acquired along the black line. In the ABF line profile, image contrast of the dark dots is inverted and displayed as peaks. 

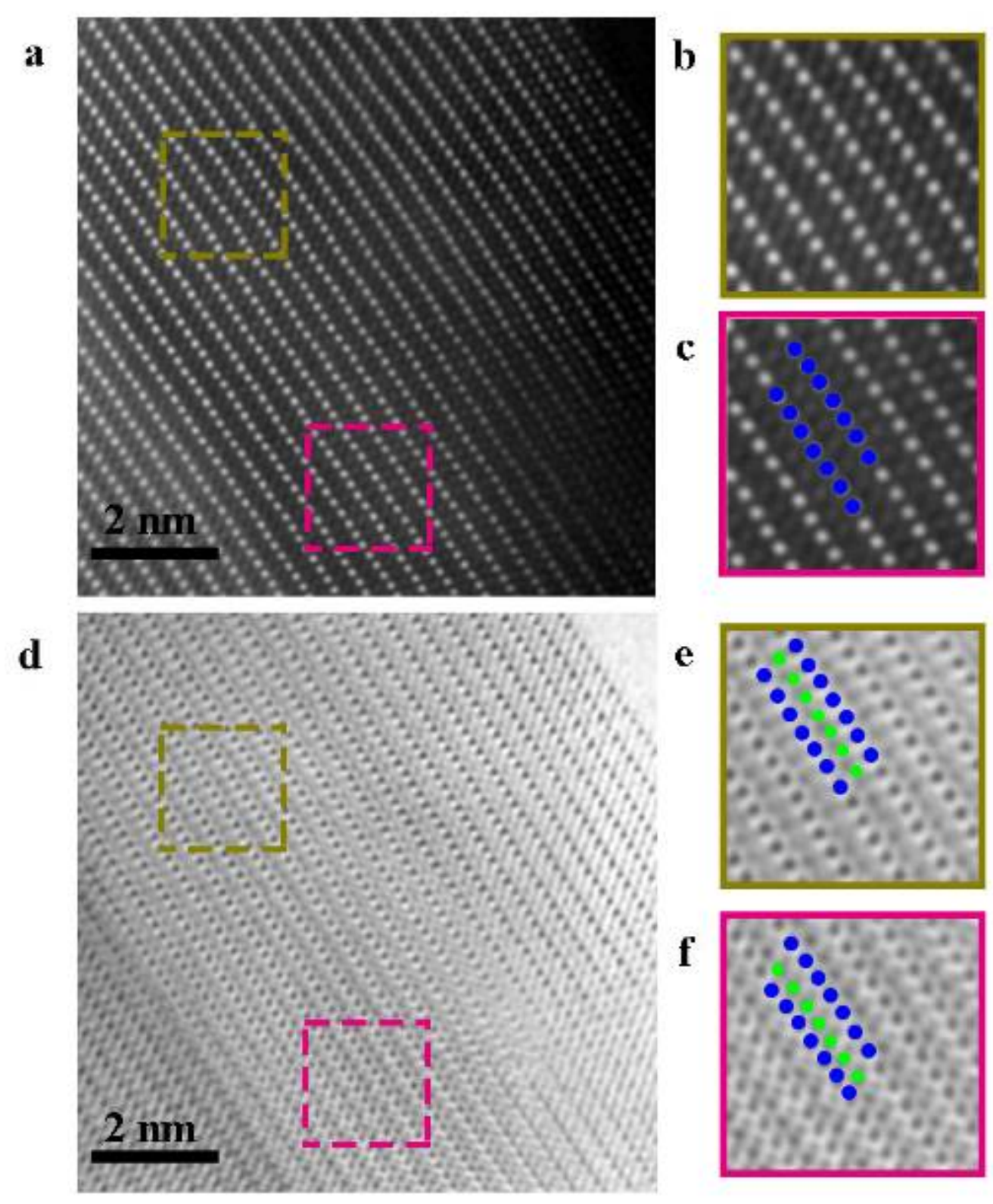

Figure 4. Structure of half charged NMC at 30C rate. a) Typical HAADF image taken along the [110] zone axis of the NMC electrode after 55 s charging at the current rate of 30C. b,c) The zoom-in image of the areas marked with orange and pink squares, respectively. d) Corresponding ABF images of NMC electrode after 55 s charging. e,f) The zoom-in image of the areas marked with orange and pink squares, respectively. The blue and green dots indicate the TM ions and Li ions, respectively. 

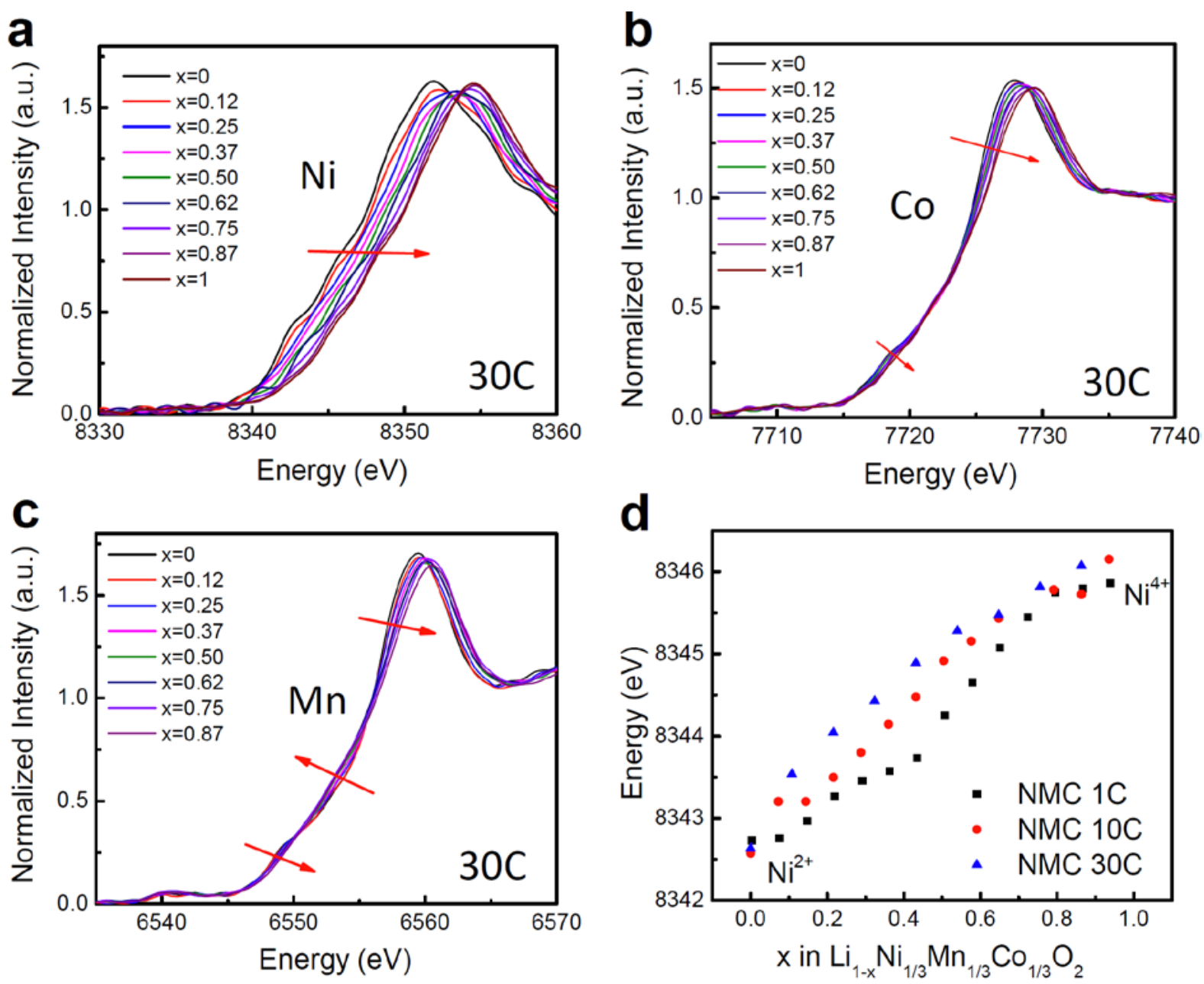

Figure 5. In situ Quick XAS of NMC during the first charge. The x-ray absorption near edge spectroscopy (XANES) results of NMC at a) Ni, b) Co and c) Mn K-edges during 30C charge, respectively. d) The Ni K-edge energy shift as a function of nominal lithium content $\mathrm{x}$ in NMC during initial charge process at the current rates of $1 \mathrm{C}, 10 \mathrm{C}$ and $30 \mathrm{C}$. 


\section{Table of Content}

Intermediate phases are revealed in $\mathrm{LiNi}_{1 / 3} \mathrm{Co}_{1 / 3} \mathrm{Mn}_{1 / 3} \mathrm{O}_{2}$ cathode during charge at $10 \mathrm{C}$ rate, and become more pronounced at $30 \mathrm{C}$ and $60 \mathrm{C}$. Li tetrahedral site occupation becomes possible when high rates are applied. The charge compensation contribution by $\mathrm{Ni}$ ions is faster and more effective at higher $\mathrm{C}$ rate, suggesting the benefit of higher Ni content in NMC for rate capability.

Keyword: lithium ion batteries, cathode materials, high rate, structure change

Yong-Ning Zhou, Ji-Li Yue, Enyuan Hu, Hong Li, Lin Gu*, Kyung-Wan Nam, Seong-Min Bak, Xiqian Yu, Jue Liu, Jianming Bai, Eric Dooryhee, Zheng-Wen Fu*, Xiao-Qing Yang*

High-Rate Charging Induced Intermediate Phases and Structural Changes of LayerStructured Cathode for Lithium-Ion Batteries

ToC figure
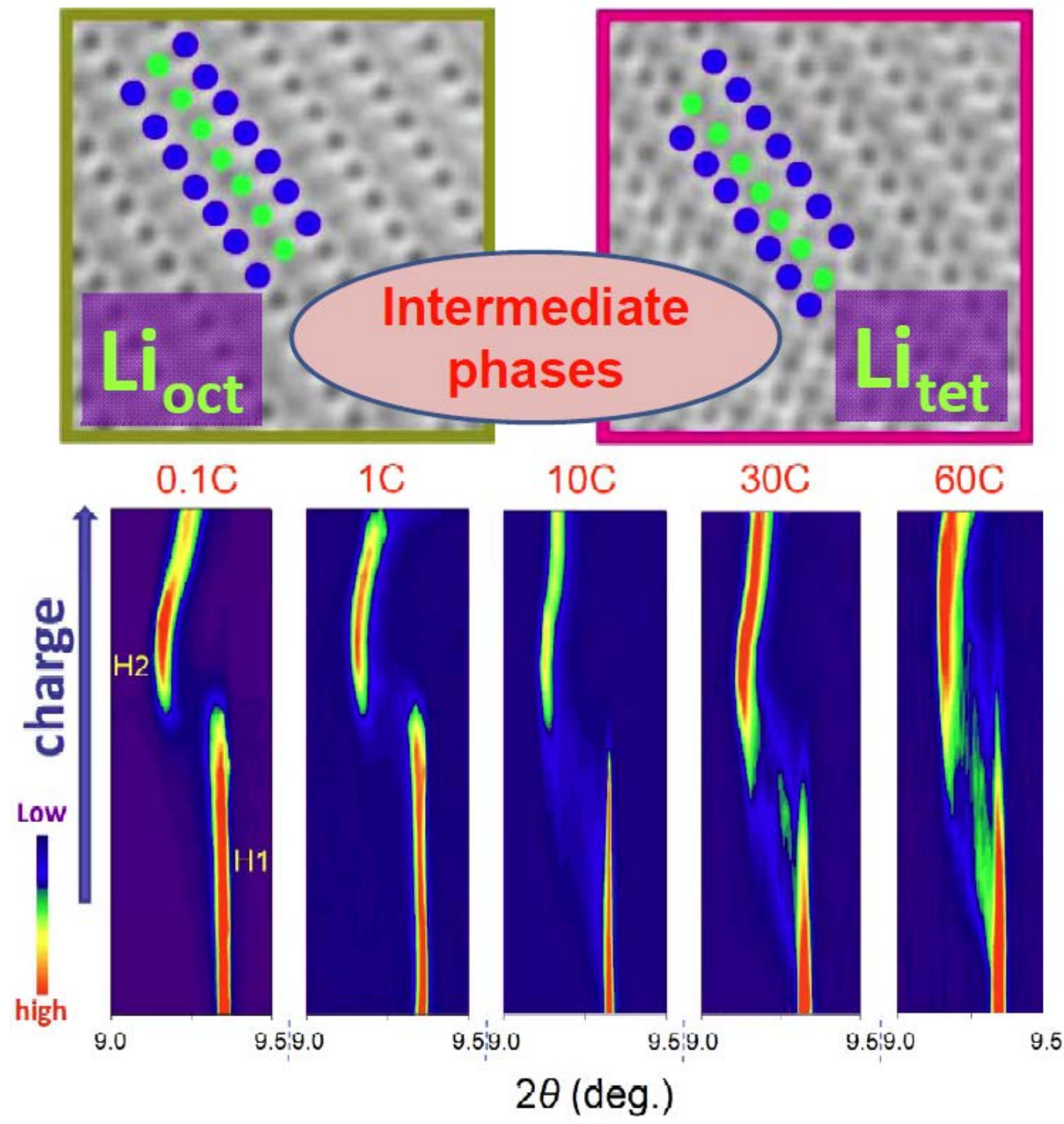

Copyright WILEY-VCH Verlag GmbH \& Co. KGaA, 69469 Weinheim, Germany, 2013. 


\section{Supporting Information}

High-Rate Charging Induced Intermediate Phases and Structural Changes of LayerStructured Cathode for Lithium-Ion Batteries

Yong-Ning Zhou, Ji-Li Yue, Enyuan Hu, Hong Li, Lin Gu*, Kyung-Wan Nam, Seong-Min Bak, Xiqian Yu, Jue Liu, Jianming Bai, Eric Dooryhee, Zheng-Wen Fu*, Xiao-Qing Yang*

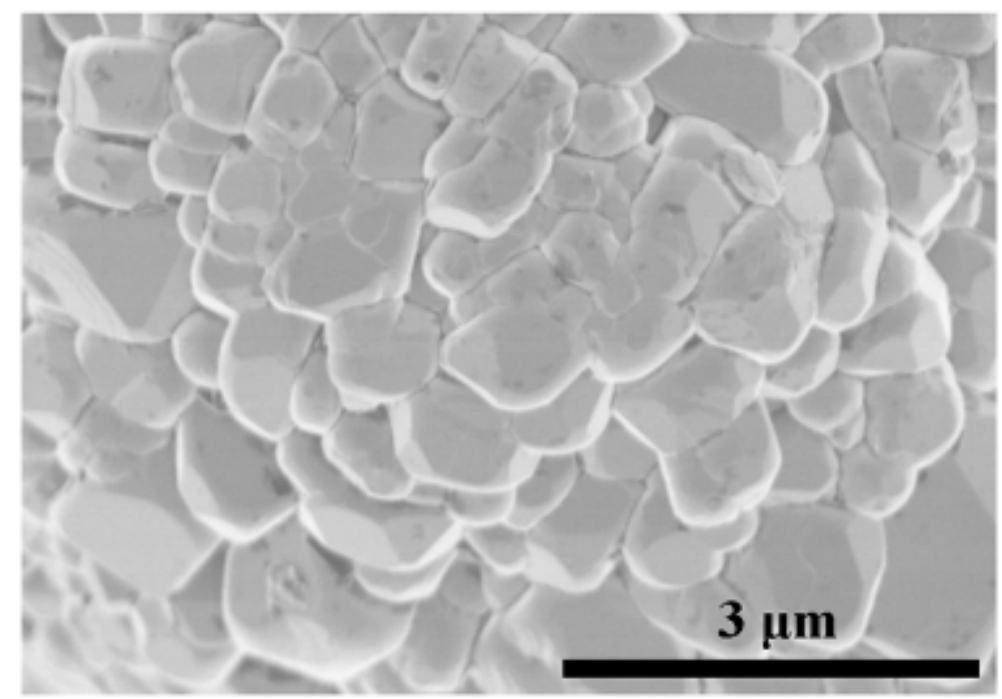

Figure S1. SEM image of pristine $\mathrm{LiNi}_{1 / 3} \mathrm{Mn}_{1 / 3} \mathrm{Co}_{1 / 3} \mathrm{O}_{2}$. Particle size is in the range of 0.8-1.0 $\mu \mathrm{m}$. 


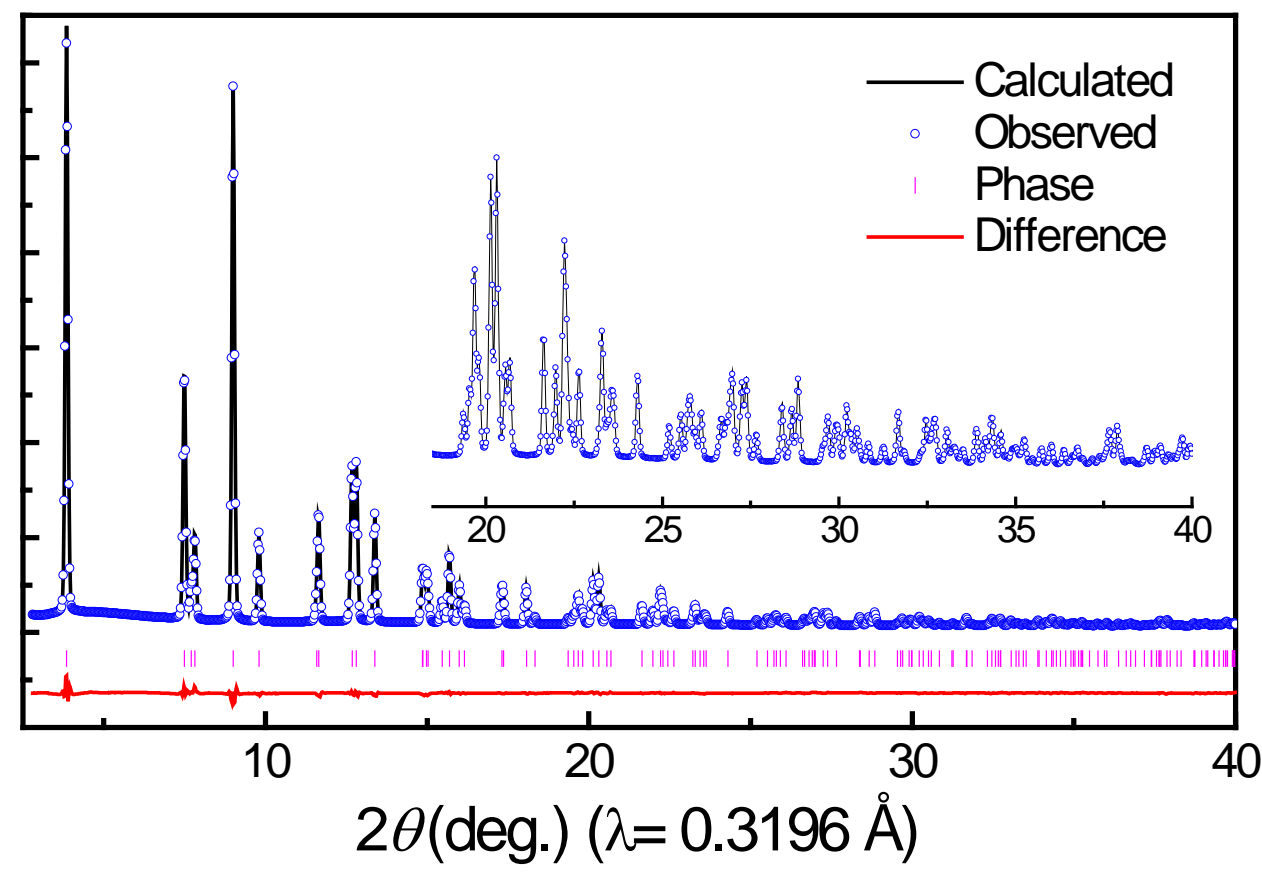

Figure S2. Rietveld refinement of the synchrotron X-ray diffraction pattern of the pristine $\mathrm{LiNi}_{1 / 3} \mathrm{Co}_{1 / 3} \mathrm{Mn}_{1 / 3} \mathrm{O}_{2}$. The XRD pattern was collected at beamline X7B of NSLS with a X-ray wavelength of $0.3196 \AA$. The experimental (blue dots) and the calculated (black line) patterns are in good agreement as shown by the flat difference curve (red line). The detailed structure information is shown in Table S1. 


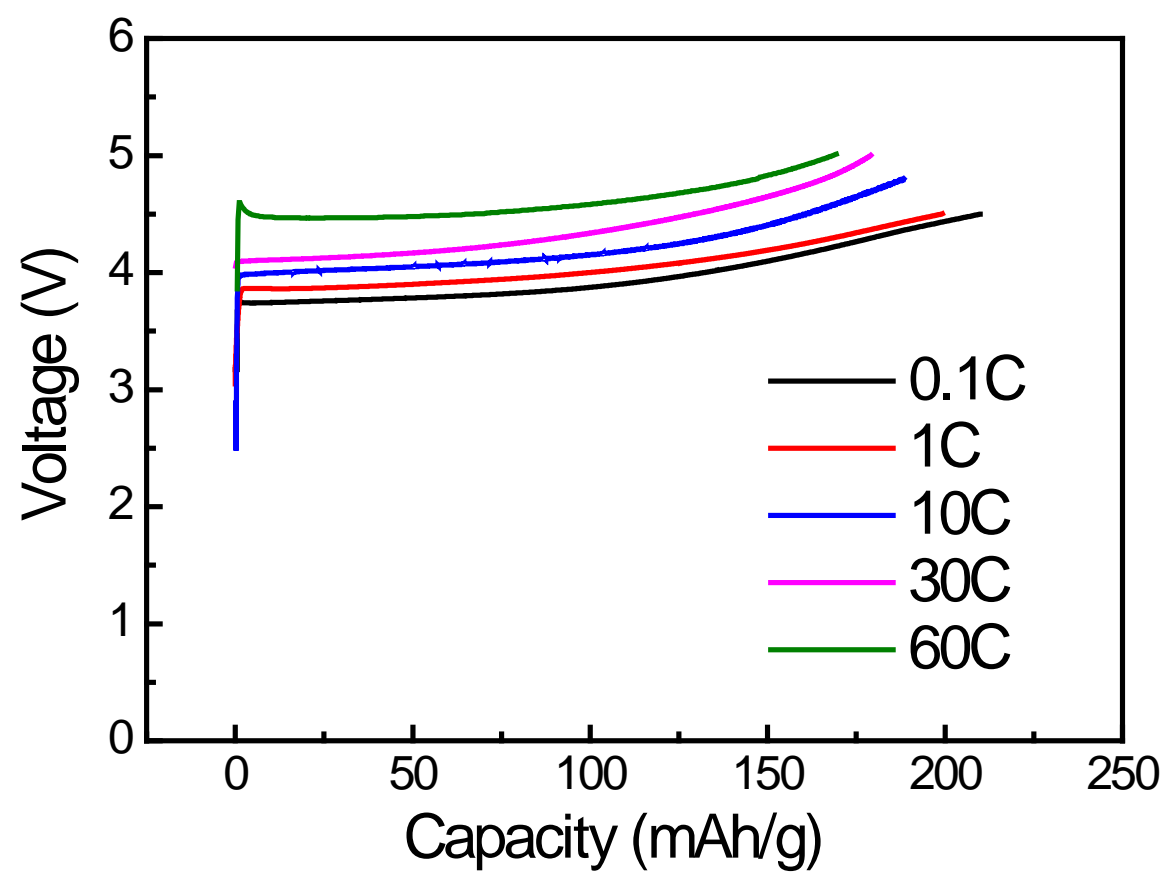

Figure S3. Charge curves of $\mathrm{LiNi}_{1 / 3} \mathrm{Co}_{1 / 3} \mathrm{Mn}_{1 / 3} \mathrm{O}_{2}$ during the in situ XRD experiment at different $\mathrm{C}$ rates. The current density at different $\mathrm{C}$ rate is calculated based on a capacity of $160 \mathrm{mAh} \mathrm{g}^{-1}$. 


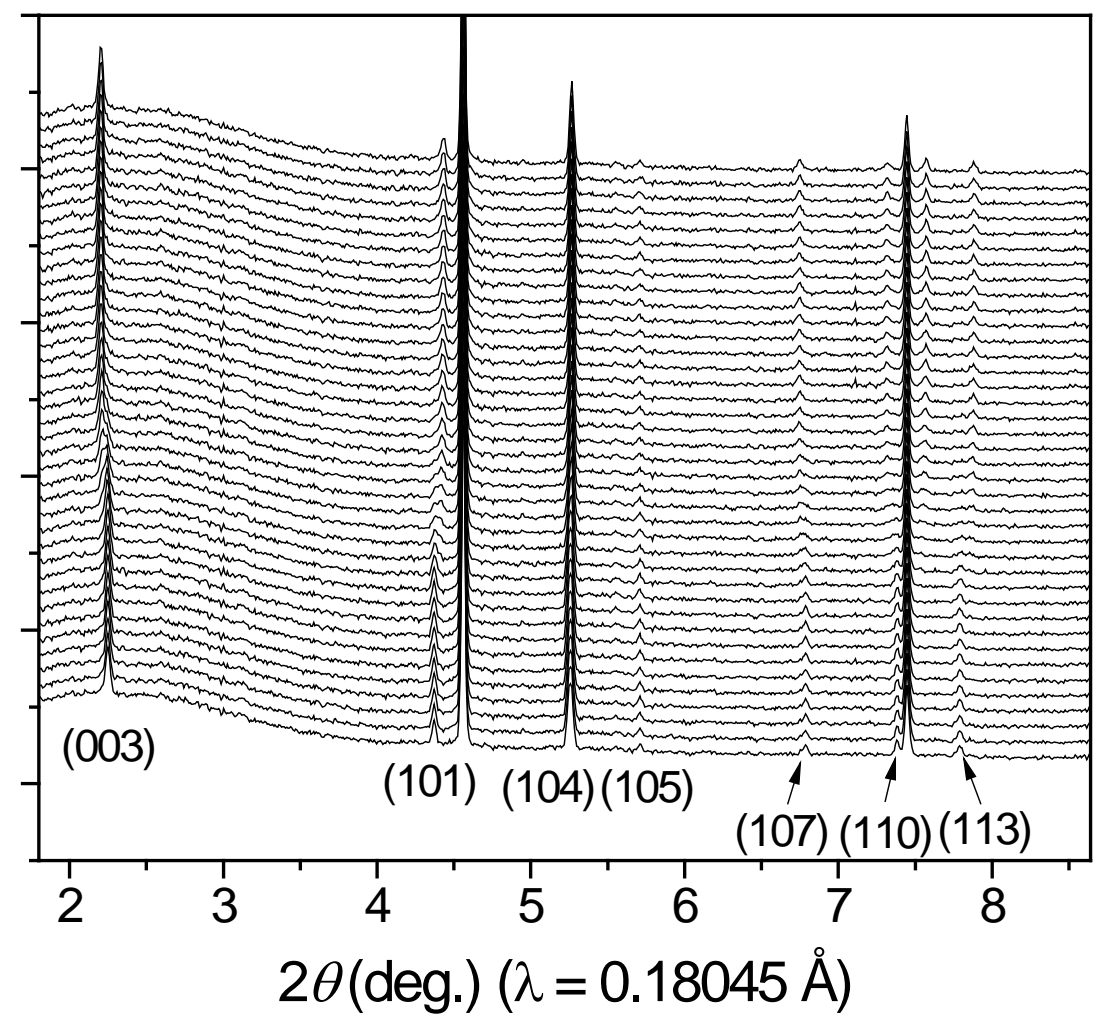

Figure S4. Full XRD patterns of $\mathrm{LiNi}_{1 / 3} \mathrm{Co}_{1 / 3} \mathrm{Mn}_{1 / 3} \mathrm{O}_{2}$ during $30 \mathrm{C}$ charging. The wavelength of the X-ray is $0.18045 \AA$. Data were collected at XPD at the NSLS-II. 


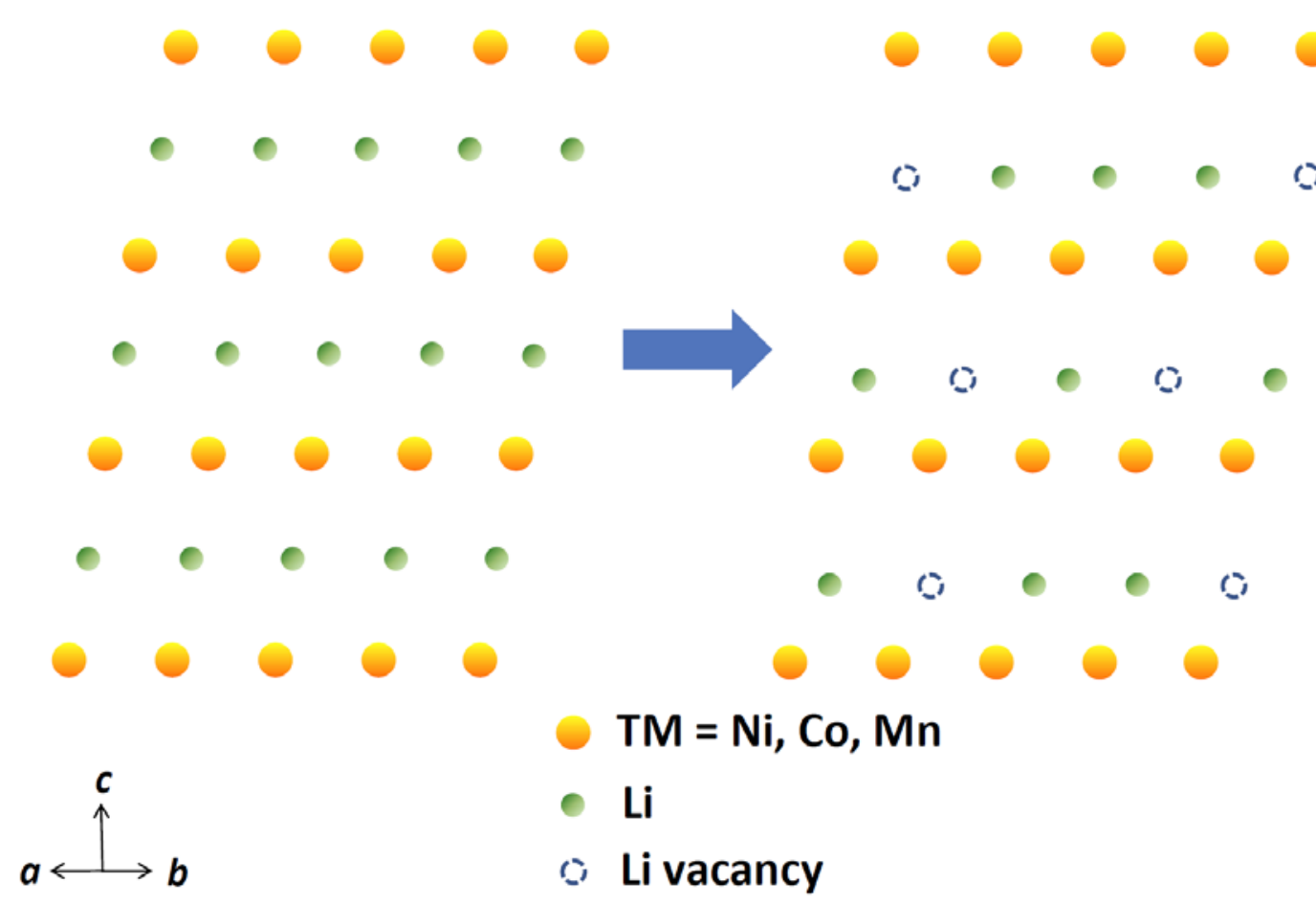

Figure S5. The structure schematic of the Li layer relocation from octahedral site to tetrahedral site along [100] projection in $\mathrm{LiNi}_{1 / 3} \mathrm{Mn}_{1 / 3} \mathrm{Co}_{1 / 3} \mathrm{O}_{2}$. 

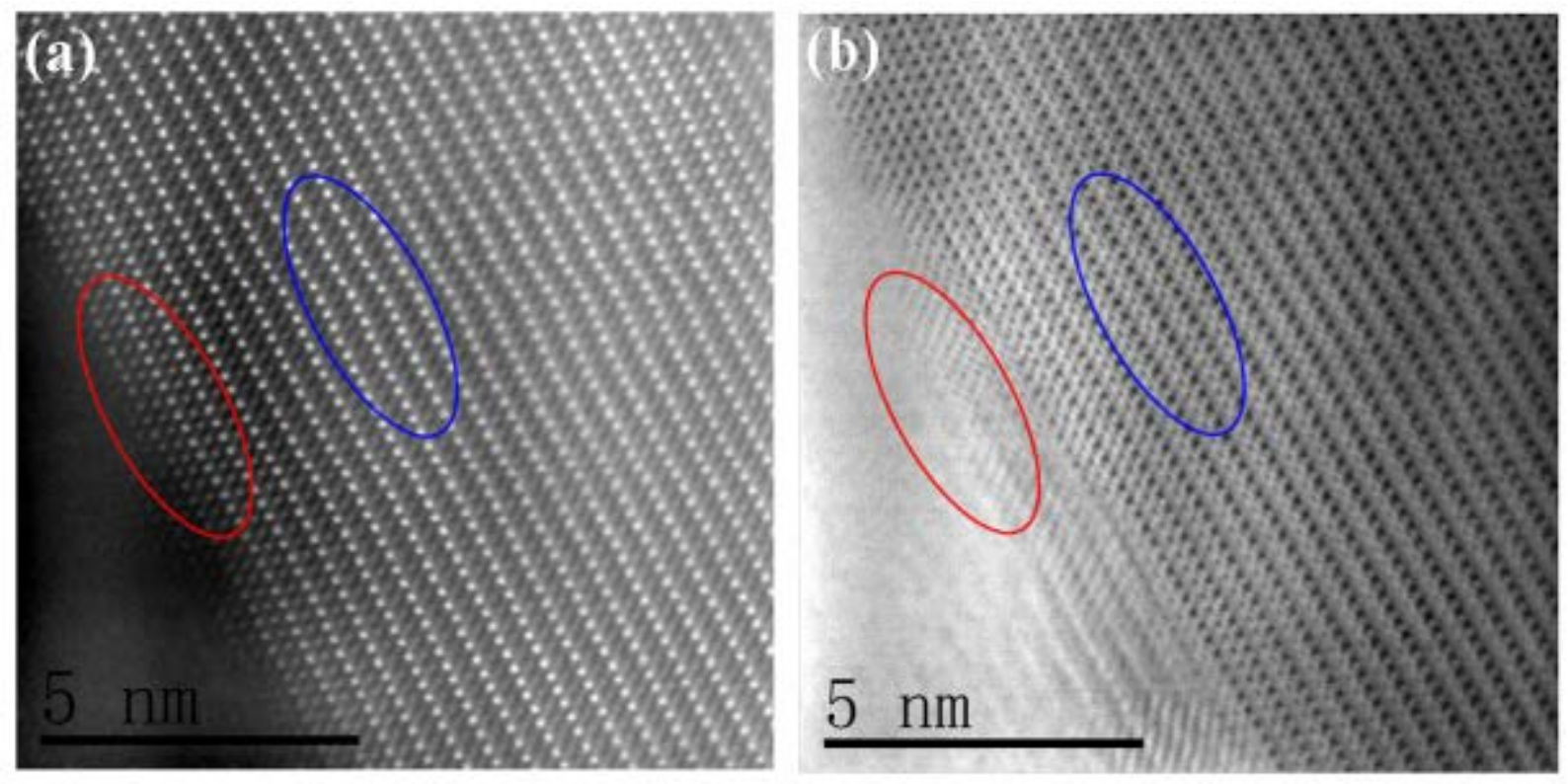

Figure S6. Atomic arrangement of $\mathrm{Li}_{1-x} \mathrm{Ni}_{1 / 3} \mathrm{Mn}_{1 / 3} \mathrm{Co}_{1 / 3} \mathrm{O}_{2}$ at the full charged state $(x=0.7)$. HAADF (a) and ABF (b) images of fully charged $\mathrm{LiNi}_{1 / 3} \mathrm{Mn}_{1 / 3} \mathrm{Co}_{1 / 3} \mathrm{O}_{2}$ at the current rate of 30C. HAADF (a) reveals some Li-TM antisites in the surface region. And in the ABF image (b), the Li ions are all located in the middle of the TM layer. Comparing with the well preserved structure in the blue circles in bulk, some Li-TM anti-site occupation (3a and 3b sites) can be observed at the surface region in the red circles. 

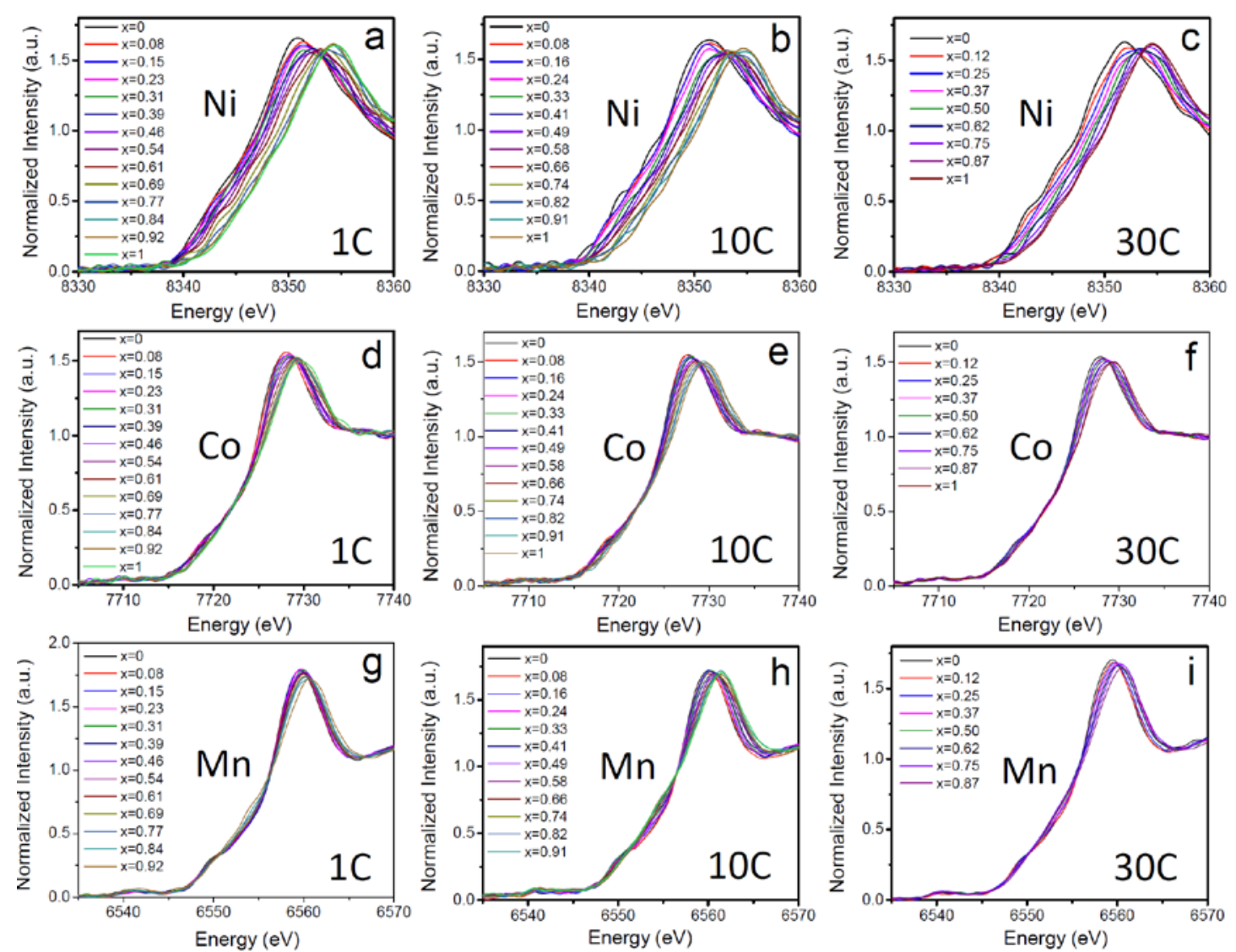

Figure S7. In situ Quick XAS of $\mathrm{LiNi}_{1 / 3} \mathrm{Mn}_{1 / 3} \mathrm{Co}_{1 / 3} \mathrm{O}_{2}$ during the first charge at different $\mathrm{C}$ rate. The Ni (a, b, c), Co (d, e, f) and Mn (g, h, i) K-edge measured as a function of lithium content in NMC during the initial charge at the current rates of 1C, 10C and 30C, respectively. $x$ value in the figure legends indicates the number of $\mathrm{Li}$ deintercalated per $\mathrm{Li}_{1}$ ${ }_{x} \mathrm{Ni}_{1 / 3} \mathrm{Mn}_{1 / 3} \mathrm{Co}_{1 / 3} \mathrm{O}_{2}$. 

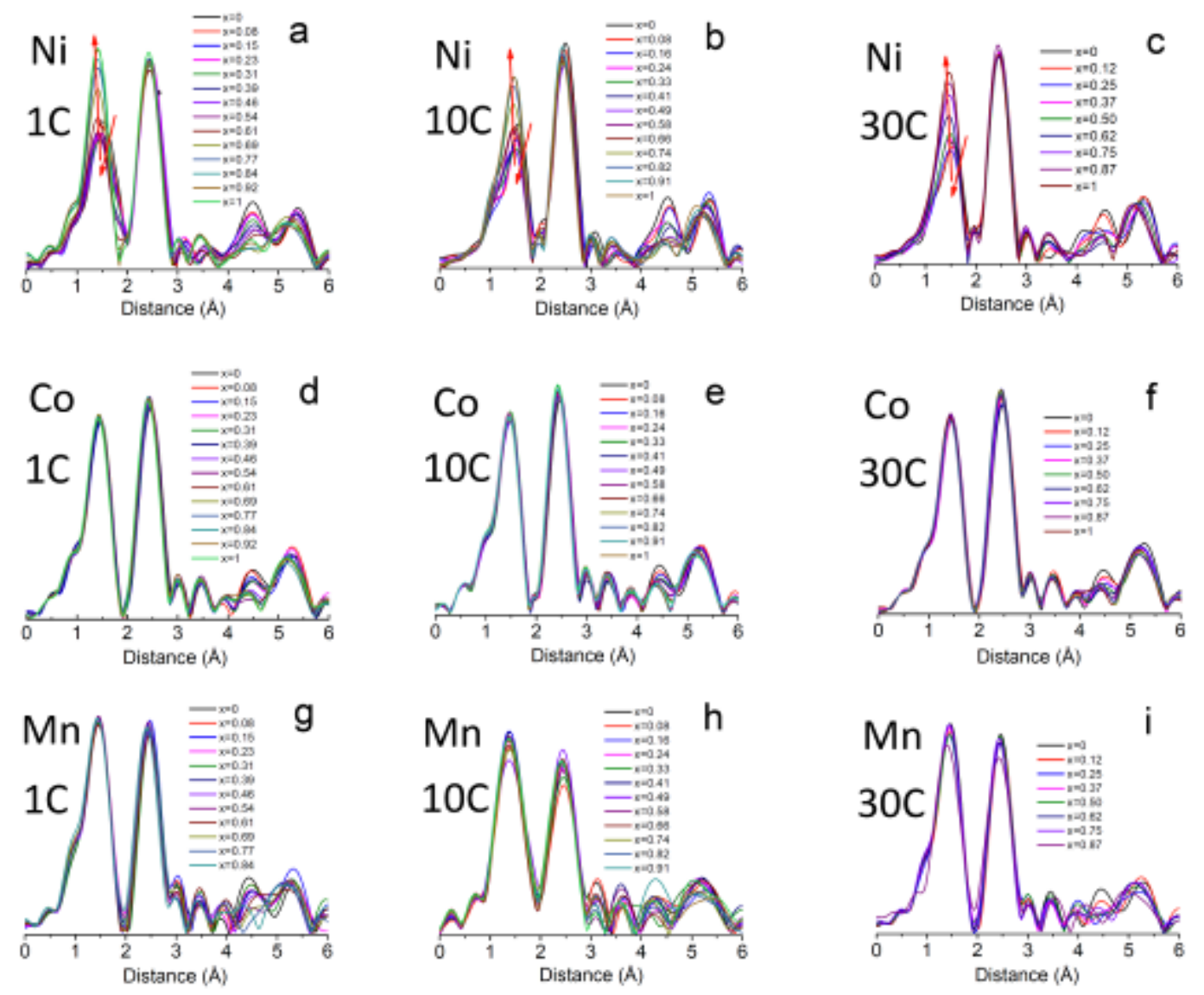

Figure S8. In situ FT-EXAFS spectra of NMC at different C rates. The Fourier-transformed extended X-ray absorption fine structure (FT-EXAFS) spectra for Ni (a,b,c), Co (d,e,f) and $\mathrm{Mn}$ (g,h,i) K-edges $\left(k^{3}\right.$-weighted in $k$-space) of $\mathrm{Li}_{1-x} \mathrm{Ni}_{1 / 3} \mathrm{Mn}_{1 / 3} \mathrm{Co}_{1 / 3} \mathrm{O}_{2}$ during initial charge at the current rates of 1C, 10C and 30C. $x$ value in the figure legends indicates the number of $\mathrm{Li}$ deintercalated per $\mathrm{Li}_{1-x} \mathrm{Ni}_{1 / 3} \mathrm{Mn}_{1 / 3} \mathrm{Co}_{1 / 3} \mathrm{O}_{2}$. 
Table S1. Structural parameters of NMC. The refined (by GSAS program) structural parameters of the as-prepared $\mathrm{LiNi}_{1 / 3} \mathrm{Co}_{1 / 3} \mathrm{Mn}_{1 / 3} \mathrm{O}_{2} \mathrm{H} 1$ phase from the synchrotron $\mathrm{X}$-ray diffraction data (experimental pattern and fit are shown in Figure S2).

\begin{tabular}{|c|c|c|c|c|c|c|}
\hline Atom & Position & $x / a$ & $y / b$ & $z / C$ & Occup & $U_{\text {iso }}\left(\AA^{2}\right)$ \\
\hline \multicolumn{7}{|c|}{$\mathrm{LiNi}_{1 / 3} \mathrm{Co}_{1 / 3} \mathrm{Mn}_{1 / 3} \mathrm{O}_{2}(\mathrm{R}-3 \mathrm{~m}): a=b=2.8618(3) \AA, c=14.2450(4) \AA$} \\
\hline $\mathrm{Li}$ & 3a & 0.000000 & 0.000000 & 0.000000 & 0.987 & 0.00623 \\
\hline $\mathrm{Ni}(1)$ & За & 0.000000 & 0.000000 & 0.000000 & 0.013 & 0.00623 \\
\hline $\mathrm{Ni}(2)$ & $3 b$ & 0.000000 & 0.000000 & 0.500000 & 0.313 & 0.00326 \\
\hline Co & $3 b$ & 0.000000 & 0.000000 & 0.500000 & 0.335 & 0.00326 \\
\hline Mn & $3 b$ & 0.000000 & 0.000000 & 0.500000 & 0.338 & 0.00326 \\
\hline $\mathrm{O}$ & $6 c$ & 0.000000 & 0.000000 & $0.240845(0.000049)$ & 0.976 & 0.00650 \\
\hline
\end{tabular}


Table S2. Lattice parameters measured from the STEM images.

\begin{tabular}{lll}
\hline Phase & $a(\AA)$ & $c(\AA)$ \\
\hline pristine NMC & 2.85 & 14.21 \\
half charged NMC (lithium rich phase) & 2.82 & 14.30 \\
half charged NMC (lithium poor phase) & 2.79 & 14.46 \\
\hline
\end{tabular}

